\title{
Adaptation of control structure design methods to an industrial plant engineering workflow
}

\author{
S. Hoeser ${ }^{\mathrm{a}}$, S. Arora ${ }^{\mathrm{b}}$, M. Koitka ${ }^{\mathrm{a}}$, T. Kleinert ${ }^{\mathrm{c}}$, O. Kahrs $^{\mathrm{b}}$, D. Sebastia-Saez ${ }^{\mathrm{d}}$, \\ H. Arellano-García ${ }^{\mathrm{d}, *}$ \\ ${ }^{a}$ BASF SE, Automation Technology, D-67056 Ludwigshafen, Germany \\ ${ }^{b} B A S F$ SE, Corporate Operational Excellence, D-67056 Ludwigshafen, Germany \\ ${ }^{c} B A S F$ Antwerpen N.V., Technology Polyurethanes, B-2004 Antwerpen, Belgium \\ ${ }^{d}$ Department of Chemical and Process Engineering, University of Surrey, Stag Hill Campus, \\ GU2 7XH, Guildford, United Kingdom
}

\begin{abstract}
Methods of systematic control structure design are adapted to suit industrial workflow of plant design and engineering in the chemical and process industry. The applied methods include a systematic step-wise design methodology and the concept of self-optimizing control. Implementation of these methods is performed in a commercial software framework that consists of Aspen Plus ${ }^{\circledR}$ and MATLAB ${ }^{\circledR}$. The control structure design methodology of a sequence of heat-integrated distillation columns is presented as a case study.

Keywords: Industrial workflow, Control structure design, Plantwide control, Self-optimizing control, Controlled variable selection
\end{abstract}

\section{Introduction}

The design of a chemical plant substantially determines its economic competiveness. Advanced process designs increasingly demand advanced control designs to ensure stable operability and to optimize plant operation. For example, extensive energy and material recycle streams improve energy and raw-material efficiency, but lead to more widespread effects of local control actions. Downsiz-

\footnotetext{
This document is a collaborative effort.

*Corresponding author

Email address: h.arellano-garcia@surrey.ac.uk (H. Arellano-García)
} 
ing and neglecting buffer tanks reduce investment cost, but complicate damping of process disturbances. Integrated unit operations such as divided-wall columns and reactive distillation columns reduce investment costs, but provide fewer control valves compared to the corresponding non-integrated units.

The conceptual design of continuously operated plants is often based on steady-state process models. The dynamic process behavior is often anticipated by experienced engineers or is derived from pilot plants and similar plants already in operation. If considered necessary, dynamic models are created for simulated operability studies of transient process steps (e.g. start-up, loadchanges), controller design, and training simulators. Otherwise, controller tuning as well as control structure improvement is done during plant operation.

As sophisticated control and optimization strategies using the advantages of MIMO control methods (e.g. model predictive control, real-time optimization) are usually not available for plant commissioning and for the first years of operation, the control design task of pairing controlled and manipulated variables of the single-loop controllers is important. Thus, the optimal pairing for anticipated operating and disturbance scenarios is a challenging task. To this end, the authors investigate the control structure design method of Skogestad ${ }^{1}$ for its applicability in an industrial environment. This method promises to meet industrial requirements of low additional modeling effort, robustness and numerical efficiency. This article provides a concept for its integration in an industrial plant design workflow to acknowledge and guide development activities of academia and vendors of plant design tools.

The paper is organized as follows: Section 2 summarizes the control structure design method ${ }^{1}$ and its implementation using two commercial software packages for steady-state process modeling (Aspen Plus $\left.{ }^{\circledR}\right)$ ) and data analysis $\left(\right.$ MATLAB $\left.^{\circledR}\right)$. A heat-integrated distillation train is selected as case study and presented in Section 3. Simulation results are discussed in Section 4 and the applicability of the control structure design method in an industrial environment is discussed. Section 5 summarizes the key results of this study. 


\section{Methodology}

Control structure selection for complete chemical plants is a topic of research for several years now. Two kinds of approaches can be found in the literature: heuristic-based and optimization-based. Heuristic-based approaches are decomposing the plantwide control problem into a number of less complex subproblems, which are dealt with in a sequential manner. Major contributions supporting this approach $\operatorname{are}^{2 \sqrt{2 / 3}}$ and collections of heuristics can be found in literature, e.g.415/6]. These control structures often work sufficiently well, but better performing alternatives might exist. In contrast, optimization-based approaches are intended to formalize the plantwide control problem into a mathematical structure which usually includes some kind of steady-state or dynamic process model. Optimization algorithms are then used to identify the optimal

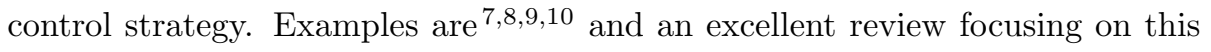
approach is given by Biegler and Grossmann 11 . A major challenge of this approach is to find a complete mathematical formulation of the plantwide control problem both because of its size and complexity. Extensive and comprehensive reviews are available which give an overview of the immense diversity of possible design strategies $12|13| 14|15| 16$.

In this work, a stepwise control structure design methodology is used, which combines the results from mathematical optimization together with heuristics. It resembles the design procedure presented by Skogestad ${ }^{1]}$ and has its roots in the work of Morari et al.17. The overall target of the design procedure is the synthesis of a hierarchical control structure with multiple control layers, i.e. supervisory and regulatory control, as established by Skogestad ${ }^{1}$. The layers are distinguished by their task and the time-scale they act upon: The upper layers influence the plant operation by adjusting setpoints for the lower layers. Primary controlled variables are directly responsible for the economic performance of a plant and are usually controlled in the supervisory layer. Secondary variables are controlled in the regulatory layer for stabilization and local disturbance rejection, respectively. The design procedure is generally iterative and may 
require several loops through the steps before converging to a valid control structure.

[Table 1. about here]

The methodology consists of two major parts. Steps 1-4 are a systematic top-down analysis of the process, while in steps 5-8 a bottom-up design of a hierarchical control structure is conducted:

Step 1. Definition of operational objectives and process constraints $g$

Step 2. Control degree of freedom analysis and selection of manipulated variables $u$

Step 3. Selection of primary controlled variables $c=y_{1}$ and pairing

Step 4. Design of throughput control

Step 5. Selection of secondary controlled variables $y_{2}$ and design of regulatory layer

Step 6. Design of supervisory layer

Step 7. Design of optimization layer (e.g. real-time optimization or model predictive control layer)

Step 8. Dynamic validation

Major advantages of this approach are that the method is fairly intuitive and focuses on optimal economic plant operation, while trying to keep the control structure as simple as possible (preferably single-input single output controllers with constant setpoints). Controlled variable sets selection (step 3) is strictly systematic (see Section 2.1) and its basic principles have been tested successfully in industrial applications ${ }^{18}$. Screening criteria (see Section 2.2 for the search of promising controlled variables are also available. Moreover, despite the fact that the search process represents a combinatorial problem, it can be solved with low computational effort (see Section 2.3). Ultimately, the design process is completely based on steady-state models, which allows efficient reuse of existing plant models that are developed in almost all new investment projects during the plant design process. The methodology has therefore the capability of narrowing the gap between model-based process design and model-based control design (step 7), which still prevents the implementation of many sophisticated model- 
based control strategies ${ }^{19}$. Details on the controlled variable selection strategy are given in the following subsections, starting with a short introduction of the concept of self-optimizing control. This work focuses on highlighting the main ideas and references are given.

\subsection{Selection of primary controlled variables}

Every chemical plant is subject to operational objectives, such as the minimization of energy usage or the maximization of throughput. In order to accomplish this task, the manipulated variables $u$ (e.g. cooling and heating duties, mass flows) need to be optimized and adjusted in the presence of disturbances $d$ (e.g. changes in raw material composition, ambient temperature, steam pressure loss). In eq. 1 the objective function $J$ represents the operational objectives which can be minimized by adjusting the manipulated variables $u$. The optimization therefore seeks the optimum value of $u(d)[20]$ :

$$
\min _{u} J(u, d)=J\left(u_{o p t}(d), d\right)=J_{o p t}(d)
$$

The optimization is subject to the constraints:

$$
\begin{aligned}
& g_{1}(u, d)=0 \\
& g_{2}(u, d) \leq 0
\end{aligned}
$$

Inequality constraints $g_{2}$ are some limitations (or specifications) on the process, which need to be satisfied in order to stay in a feasible region while equality constraints $g_{1}$ include the model equations. It is frequently observed that some inequality constraints are "active" at the optimum $\left(g_{2}=0\right)$ for the whole disturbance space D. It is therefore straightforward to choose controlled variables c and constant setpoints $c_{s}$ such that $c=g_{2}$ and $c_{s}=0$. A popular example are top and bottom product compositions of distillation columns which are always at their respective specifications in energy optimal operation: it is $g_{2}=w_{t o p}^{l}-w_{t o p}=0$ at the optimum, with $w_{t o p}$ being the top composition and $w_{\text {top }}^{l}$ being the top product specification. Consequently, the controlled variable is $c=w_{t o p}^{l}-w_{t o p}$ and the constant setpoint should be chosen as $c_{s}=0$. 
With the active constraints controlled, the remaining degrees of freedom are unconstrained and adding control laws with constant setpoints might move the operation far away from optimal in the presence of disturbances $(J(u, d) \neq$ $\left.J_{\text {opt }}(d)\right)$. Hence, it is necessary to find controlled variables $c$, which when kept at constant nominal setpoints $c_{s}^{*}$ minimize the imposed loss $L$. This concept is called "self-optimizing control" 20, where the loss $L$ quantifies the nearoptimality of self-optimizing control structures. It is defined as the decrease in profitability compared to optimal control $J_{o p t}$ :

$$
L=J(u, d)-J_{o p t}(d)
$$

The loss $L$ is caused by a disturbance-dependent setpoint error $\nu(d)$ for the unconstrained degrees of freedom

$$
\nu(d)=c_{s}-c_{o p t}(d)
$$

and a controlled variable related implementation error $n$ due to poor instrumentation (e.g. measurement noise):

$$
n=c-c_{s}
$$

In step 3 the selection procedure of primary controlled variables with selfoptimizing properties consists of several sub-steps:

sub-step 3.1 Specify the expected disturbances $d$ and implementation errors $n$

sub-step 3.2 Minimize $J(u, d)$ to find $J_{\text {opt }}(d)$

sub-step 3.3 Identify and control active constraints

sub-step 3.4 Screen for promising candidate controlled variable sets

sub-step 3.5 Exact nonlinear evaluation of loss $L$

sub-step 3.6 Select a promising set of controlled variables

The basic concept of candidate controlled variable screening (sub-step 3.4) is presented in the next subsection. 


\subsection{Screening criteria}

Exhaustive evaluation of the loss $L$ using nonlinear process simulations (substep 3.5) for all possible sets of controlled variables is computationally inviable, and thus, an effective screening is desirable (sub-step 3.4). Screening methods usually introduce simplifications and that is why their results are to be regarded as proposals. From the former section, it can be concluded that a process variable suitable for self-optimizing control should satisfy the following qualitative requirements:

1. Its optimal value $c_{\text {opt }}(d)$ should be relatively insensitive to disturbances $(v(d) \rightarrow 0$, cf. Eq. 3).

2. It should be easy to measure and to control $(n \rightarrow 0$, cf. Eq. 4). Additionally,

3. Good controlled variables $c$ are sensitive to changes in the manipulated variables $u$ and disturbance variables $d$.

4. For decentralized control, it is recommended to minimize the interaction between the individual closed control loops.

In order to automate the screening, the requirements must be transformed into a quantitative measure. A simple and effective approach for loss estimation $L_{e s t}$ is based on a local second order Taylor series expansion around the optimal nominal operating point 21 :

$$
L_{e s t}=\frac{1}{2}\|z\|_{2}^{2}
$$

with $z=J_{u u}^{1 / 2} G^{-1} e_{c}$.

The estimated loss $L_{e s t}$ can thus be expressed using the steady-state gain matrix $G$ with $G_{i, j}=\frac{\partial c_{i}}{\partial u_{j}}$, the objective function Hessian $J_{u u}$ with $\left[J_{u u}\right]_{i, j}=$ $\frac{\partial^{2} J}{\partial u_{i} \partial u_{j}}$, and the control error $e_{c}=n+\nu(d)$.

It can be seen that this formulation incorporates the first three of the requirements mentioned above (1-3): The less $L_{e s t}$ is reduced, the more they are met. 
Two different approaches to express the control error $e_{c}$ have been proposed: the minimum singular value rule (MSV rule) 21 , which incorporates the maximum setpoint error $\max _{d}(\nu(d))$ obtained from nonlinear steady-state simulations of a set of discrete disturbance scenarios, and the exact local method (ELM) which linearizes the process model around the optimal nominal operating point in order to express the setpoint error directly dependent on the disturbance variables $d^{22}$,

$$
\nu(d)=\left(G J_{u u}^{*-1} J_{u d}^{*}-G_{d}\right)\left(d-d^{*}\right)=M_{d} d^{\prime},
$$

where $J_{u d}$ is the objective function Hessian $\left[J_{u d}\right]_{i, j}=\frac{\partial^{2} J}{\partial u_{i} \partial d_{j}}$ and $G_{d}$ is the steady-state disturbance gain matrix $\left[G_{d}\right]_{i, k}=\frac{\partial c_{i}}{\partial d_{k}}$. The control error $e_{c}$ can then be expressed as

$$
e_{c}=\left(M_{d} M_{n}\right)\left(\begin{array}{l}
d^{\prime} \\
n^{\prime}
\end{array}\right)=M\left(\begin{array}{l}
d^{\prime} \\
n^{\prime}
\end{array}\right)
$$

with $M_{d}$ and $M_{n}$ as weight matrices and $d^{\prime}$ and $n^{\prime}$ being normalized to have magnitudes less than 1 . From both forms the estimated worst-case loss $L_{e s t}$ can be evaluated using singular value decomposition instead of nonlinear process simulations. The MSV rule is intuitive and gives a good first impression while the complete linear models used in the ELM allow more detailed evaluation, e.g. contribution of a single disturbance to the estimated loss. Both criterions are simple, fast to compute and have proved to give good results ${ }^{23}$.

[Figure 2 about here]

The result of the screening is a ranking of "promising" candidate controlled variable sets based on the respective evaluation criterion (MSV rule or ELM). However, potential interaction problems are not considered at this step of the original design procedure in order to separate economic and control performance ${ }^{1}$. In practice, once the degree of freedom analysis has been performed (step 2), the selection of an independent set of manipulated variables is straightforward and required for process simulation. The steady-state gains $G=\frac{\partial c}{\partial u}$ are 
available (eq. 5) and therefore, the relative gain array (RGA; 24 ) can be calculated as simple interaction measure for the most promising candidate variable sets. This helps to identify questionable sets even before the computationally expensive evaluation of exact nonlinear loss in sub-step 3.5. Thus, both the automated screening and the RGA analysis help reducing the number of iterations required so as to design a feasible control structure.

All evaluations discussed here are based on steady-state process data only. Stabilizing control of pure integrators (e.g. liquid levels in buffer tanks) has no influence on the result of both screening methods (MSV rule, ELM or RGA analysis).

\subsection{Software framework for automated screening}

For the candidate controlled variable screening methods introduced in Section 2.2 information about the process is needed, which is obtained from the evaluation of several different operating points. In this study, the commercial process simulation software package Aspen Plus ${ }^{\circledR}$ is used. The tool offers several features, however the ease with which a steady-state simulation can be converted to dynamic simulation is quite helpful. It also provides the Aspen OOMF Script language as automation tool. This procedural programming language is based on Fortran, which features standard functionality such as mathematical and string functions, if-then-else logic and for-do loops. Moreover, Aspen OOMF script files can be created and edited using simple text editors.

However, OOMF is not powerful enough for complex matrix operations such as singular value decomposition, which is required for loss estimation. Thus, in this work the results obtained from Aspen Plus ${ }^{\circledR}$ simulations are exported to the MATLAB ${ }^{\circledR}$ software package. Furthermore, powerful branch and bound algorithms ${ }^{25|26| 27}$ are used, which greatly reduce the computational effort in comparison to the exhaustive "brute-force" search method. They are available in the Matlab File Exchange platform 2829130 .

For this software framework a package of scalable scripts (OOMF scripts and MATLAB m-files) can be created, which means then that the package can 
be applied to any properly configured Aspen Plus ${ }^{\circledR}$ simulation, provided that the user has supplied the definition of the unconstrained control problem (steps 1 to sub-step 3.3).

The basic tasks of both softwares and the data flow is illustrated schematically in Fig. 2. The interface is formed by ASCII files, which document the selection problem and make it independent of the simulation software. Simulation and optimization results as well as sensitivities and objective function

gradients can easily be exported from Aspen Plus ${ }^{\circledR}$. However, the objective function Hessian $\left(J_{u u}\right.$ and $\left.J_{u d}\right)$ is not accessible. It is therefore approximated using a finite differences approach (central difference formula, see e.g. ${ }^{31}$ ).

\section{The example process: Sequence of four heat-integrated distillation columns}

The example process is a heat-integrated distillation train for the separation of only heavy components (purification of component $\mathrm{C} 0$ ). The major fraction of the feed stream is $C 0$ and the rest of it are impurities $C 1, C 2, C 3, C 4, C 5$ and $C 6$. Due to confidentiality reasons, the original component names cannot be disclosed. Normalized property ratios of the components are given in Table 1. The reflux is very low and only used to shut down some heavy components. The packing is designed for very low liquid flow. The temperature difference of the first three columns is only due to the pressure drop and the changing boiling point of $\mathrm{C} 0$ across the column. Moreover, temperature could be used as indication of quality due to the different boiling points of the single pure components.

[Figure 3 about here]

The objective is to separate as much $C 0$ from the impurities while keeping the operating costs minimal. From Table 1, it can be seen that $C 0$ is the lightest key, and thus, the separation demands to be highly energy-consuming if the specification on the purity is extremely high, in particular, when operating with only one column. In order to increase the energy efficiency, a sequence of 
four heat-integrated distillation columns as shown in Fig. 2 is used.

[Table 2 about here]

Only a fraction of the feed is boiled up in the first column, and thus, requiring less heat. The remaining is fed to the second column, where the bottom stream of the first column represents the feed stream of the second column. The condensation heat of the first column is used as reboiler duty for the second column. Since heat is transferred along temperature gradients, a sufficient temperature difference has to be provided between the vapour of the first column and the sump of the next column. This is accomplished by operating the second column at a lower pressure than the first column $(P 1>P 2)$. This principle is used another two times in such a way that there is a sequence of four distillation columns with three times forward heat-integration with $P 2>P 3>P 4$. The heat-integration is complete: all condensation heat is consumed as reboiler duty in the successive column. There are no extra degrees of freedom such as additional heat exchangers, which could be used as heat sinks or sources in order to adjust the reboiler heat duties of each column individually.

The first three columns have total condensers while the last one uses a partial condenser. Due to the low operating pressure of the last column, the cooling water fed to the cold side of the condensing heat exchanger can only cool the vapour up to a limited extent. Thus, a fraction of the boiled up $C 0$ leaves as vapour distillate.

The liquid distillate streams of all four columns are collected in a large drum. The entire process has one feed $F$ and two major product streams: the bottom stream $B 4$ and the collected distillate $D$. At the nominal operating point, the overhead of each column is approximately one fourth of the feed mass flow $\dot{m}_{F}$.

This industrial process was chosen as a case study because of the strong thermal coupling between the columns, which makes it challenging to be controlled in an economically cost- efficient manner. There are individual, but highly integrated operational objectives e.g. product specifications on the bottom stream $B 4$ of column 4 and the overall distillate stream $D$ have to be satisfied. The operation of all four columns is closely connected, and has to be coordinated 
in order to reach the objective. Thus, a consistent inventory control structure is required to buffer throughput disturbances and dampen their influences on the quality of the separation as much as possible. In summary, the considered example process possesses several significant characteristics which render the design of its control structure an interesting and challenging plantwide control problem.

\subsection{Model implementation in Aspen Plus ${ }^{\circledR}$}

For this study, a process model was implemented in Aspen Plus ${ }^{\circledR}$ and the operation was analyzed using inbuilt process simulation and optimization routines.

[Table 3 about here]

[Table 4 about here]

The model of all four distillation columns is quite similar because of their shared purpose. The column internals are modelled as sieve trays with a given geometry, based on which the hydraulics of the internals (pressure drops, residence times, etc.) are dynamically calculated by the Aspentech software according to the simulated conditions. The calculated pressure drop over each whole column is approximately similar for all four columns, as columns with lower operating pressure are modelled having a larger diameter. Flooding calculations are conducted using the Glitsch method. The mass transfer is modeled using the equilibrium approach. Three simplifications are incorporated into the process model:

1. In order to simplify the screening procedure in step 3, some obvious control loops are closed beforehand. The top pressures of all columns $P_{1}, P_{2}, P_{3}$ and $P_{4}$ are assumed to be perfectly controlled. Since the condensers of the first three columns are specified as total condensers, this assumption renders the vapour distillate streams $V D 1, V D 2$ and $V D 3$ redundant. Consequently, they were neglected.

2. Since pressures are assumed to be perfectly controlled, the heat-integration is only modeled as a heat stream removed from the condensers and added 
to the reboiler.

3. Additionally, the reboiler of column 1 and condenser of column 4 are modeled as heat source and heat sink, respectively.

The simulations are run in the equation-oriented mode of Aspen Plus ${ }^{\circledR}$ (simultaneous solution of all equations). For dynamic simulations, the vessel volumes (reflux drums, column sumps, distillate drum) are designed such that the residence time of material is approximately $\tau \approx 5 \mathrm{~min}$ for a liquid volume fraction of $50 \%$.

\section{Problem definition and solution methodology}

Step 1: Operational objectives and process constraints. The control objective is to keep product specifications (first priority)

$$
\begin{gathered}
w_{D, C 0} \geq w_{D, C 0}^{l} \\
w_{B 4, C 0} \geq w_{B 4, C 0}^{u}
\end{gathered}
$$

while operating costs are minimized (second priority). The operating costs can be described by the following objective function $J_{1}$ :

$$
J_{1}=p_{S t} \dot{m}_{S t}+p_{C W} \dot{m}_{C W 4}
$$

where $p_{S t}$ denotes the price index for steam, $p_{C W}$ is the price index for cooling water, $\dot{m}_{S t}$ represents the steam consumption, and $\dot{m}_{C W 4}$ is the cooling water mass flow, respectively. The price for a ton of steam is set considerably higher than the price for a ton of cooling water.

The heat-integrated column sequence can be expected to behave like a single distillation column in terms of optimal operation (i.e. running at minimum operating costs) with the two active constraints (8) and (9). They should be controlled but in this case they cannot be measured online. Thus, the screening methods can be used to find substitute variables. However, the tool can only 
screen unconstrained degrees of freedom, thus, the constraints need to be translated into penalties on the objective. The bottoms product quality $w_{B 4, C 0}$ is taken as an example by using a log-barrier method, which can be implemented easily into the software framework (Aspen Plus simulation) 31 :

$$
J=J_{1} \cdot\left[1-C \cdot \lg \left(1+w_{B 4, C 0}^{u}-w_{B 4, C 0}\right)\right]
$$

The factor $C$ is tuned such that the constraint $\sqrt{9}$ is not violated in the disturbance space $D$.

A third inequality constraint limits the operational range of the partial condenser of column 4. The loss of too much $C 0$ as vapour distillate $V D 4$ is prevented by keeping the distillate temperature $T_{D 4}$ below an upper bound:

$$
T_{D 4} \leq T_{D 4}^{u}
$$

Step 2: Control degree of freedom analysis and selection of manipulated variables. Traditionally, the number of degrees of freedom $N_{D O F}$ is obtained by subtracting the sum of number of model equations $N_{e q}$ from the number of variables $N_{v}$

$$
N_{D O F}=N_{\nu}-N_{e c}
$$

Most process simulation softwares incorporate advanced features to automatically perform this task. When conducted manually, this may become cumbersome for large systems. Also, no distinction is made between control and design degrees of freedom. Therefore, several authors proposed shortcut methods, e.g.: "Count the number of control valves available" 3 or "count the number of process streams and subtract the number of extra phases" 32. In this work a different shortcut method is used to calculate the control degrees of freedom $N_{C D O F}$ 33:

" $N_{C D O F}=$ Total number of streams in that process $N_{f}$ minus the sum of the restraining numbers for all the units in that process $N_{r}$ "

where 
"Restraining number $N_{r}=$ Total number of independent and overall material balances with no associated inventory".

All units and their respective restraining number are listed in Table 3. The restraining number is increased as indicated in Table 3, if we have communicating/shared inventories between units (liquid or vapour). The overall number of streams $\mathrm{Nf}$ in the system is 47 and the total number of control degrees of freedom NCDOF yields (eq 15). However, not all of the degrees of freedom NCDOF have influence on the steady-state: nine of them are needed to control liquid levels. Additionally, the pressures are assumed to be perfectly controlled, i.e. the pressure control loops are already closed. Also, the feed is supposed to be given by upstream units and hence rather a source of disturbance than a control degree of freedom. Altogether, these considerations reduce the number of available control degrees of freedom NDOF;ss for steady-state optimization to six: (list). For plant design, this means that 20 control valves can be distributed on the lines of the case study. A line is in this case defined by a connection of inventories (e.g. the connection of the overhead vapour outlet of column 1 and the reflux drum is one line, which passes through the hot side of reboiler 2. The following rules apply: (1) Max. 1 valve per line. (2) no valves in lines that connect communicating inventories. A possible set of control valves could be distributed as listed in Table 4.. In reality, the corresponding lines, i.e. connection of inventories, should be equipped with control valves - maximum one control valve per line and no valve in lines with communication inventories. The overall number of streams $N_{f}$ in the system is 47 and the total number of control degrees of freedom $N_{C D O F}$ yields,

$$
N_{D C O F}=N_{f}-N_{r}=47-27=20
$$

They are listed in Table 3. However, not all of the listed degrees of freedom have influence on the steady-state: nine of them are needed to control liquid levels. Additionally, the pressures are assumed to be perfectly controlled, i.e. the pressure control loops are already closed. Also, the feed is supposed to 
be given by upstream units and hence rather a source of disturbance than a control degree of freedom. Altogether, these considerations reduce the number of available control degrees of freedom $N_{D O F, s s}$ for steady-state optimization to six:

\begin{tabular}{cc}
$N_{C D O F}$ & 20 \\
\hline Liquid levels & -9 \\
Pressure control loops & -4 \\
Feed given & -1 \\
\hline$N_{D O F, s s}$ & 6
\end{tabular}

sub-steps 3.1-3.3: Disturbances, optimization and active constraints. In this case study, the most important disturbances are variations of the feed properties, which include mass flow $\dot{m}_{F}$, temperature $T_{F}$, and composition $w_{F}$. Other uncertainties like pressure loss of the steam input or cooling water temperature changes are neglected. The disturbance variables $d_{i}$ and their maximum expected perturbations $\left(d^{*}-d\right)$ are listed in Table 4 .

[Table 5 about here]

[Table 6 about here]

In this case study, all temperatures, pressures, temperature differences, mass flows, heat duties, and mass flow ratios are considered as candidate controlled variables. Their maximum expected implementation errors $n_{j}$ are listed in Table 5 . Temperature and pressure implementation errors are considered constant and independent of the absolute value of the candidate controlled variable $c$. Moreover, relative implementation errors are specified for mass flows, heat duties, and mass flow ratios, respectively. Altogether, the controlled variable set needs to be selected from a pool of 133 candidates.

Although linear combinations of process variables $\mathrm{Hy}$ can be theoretically considered as controlled variable $c$ (with $H$ being the combination matrix), only single process variables $c=y$ and simple ratios of mass flows are considered as candidates in this work. The reasons are that the existing algorithms screen combinations of process variables $H y$ regardless of their physical types and that 
controlled variables in each set only differ in $H$, thus they are all combined by the same subset of process variables $y 3$ 34/35136 . Therefore, these results are usually not applicable because of low plant operator acceptance. An approach to dealing with these structural constraints has recently been proposed by 37 .

An optimal control system is aimed at adjusting the manipulated variables $u$ such that the objective function $J$ (eq. 11) is minimized for all disturbance scenarios. The results are summarized in Table 6. The optimization problem was solved for two perturbations of the eight single disturbance variables $d_{i}$. It should be noted that two process constraints are active for all disturbance scenarios: $w_{D, C 0}$ and $T_{D 4}$.

[Figure 4 about here]

Throughput variations can obviously be coped with easily if all reflux flows and the steam are adjusted proportionally to the changes of $\dot{m}_{F}$. Feed temperature disturbances are optimally rejected if the feed enthalpy difference $h_{F}(d)-h_{F}\left(d^{*}\right)$ is compensated by increased or decreased reboiler heat duty $\dot{Q}_{R B 1}$, respectively. In case of merely temperature disturbance, no change in the reflux flows of all four columns is necessary. For feed composition disturbances the following trends can be observed: If the amount of light impurities (e.g. $C 2$ ) increases then the separation is more costly and the reflux flows (or reflux ratios, respectively) as well as steam consumption need to be increased in order to comply with the process constraints. On the other hand, an increase of heavy impurities (e.g. C6) inverse observations can be made.

[Table 7 around here]

Fig. 3 shows the nominal temperature profiles of all columns. It can be seen that the temperature profile sensitivity of the first three columns to disturbances is almost negligible in comparison to the fourth column. The only column with a considerable separation is the fourth one, where the temperature difference between condenser and sump is up to 40 times the temperature difference of the first column.

[Table 8 about here]

sub-step 3.4: Candidate controlled variable screening. Four unconstrained 
degrees of freedom are left and all possible combinations of the candidate controlled variables are screened using the MSV rule and ELM as evaluation criteria. Thus, four controlled variables need to be selected out of 133 candidates thus making approximately 12.4 million possible combinations.

The screening results are shown in Table 7 (MSV rule) and Table 8 (ELM for worst-case loss minimization). The best ranked candidate controlled variable set of each screening features the reflux ratios $\nu_{1}, \nu_{2}$ and $\nu_{3}$ of the column 1-3 as well as the bottom stage temperature $T_{B 4}$ of column 4 . This seems quite reasonable, because in contrast to constant reflux-to-feed ratios $\alpha$, reflux flows $\dot{m}_{L}$ are allowed to vary in the presence of feed composition disturbances. Also, as the nominal temperature profiles in Fig. 3 show, $T_{B 4}$ is the most sensitive stage temperature of the whole column sequence. The first three columns have no significant temperature gradient in the profile and the main separation takes place in the last column. The results also show that the reflux flows of columns 1 and 2 have only negligible influence on the overall economical performance. Moreover, the worst-case loss $L_{e s t}$ is rather determined by the choice of the controlled variables associated with column 3 and 4, as highlighted by Table 7 .

[Table 9 around here]

In general, keeping stage temperatures constant in the first three columns is not promoted by any screening method. There are two reasons for that: First, their sensitivities to changes in the manipulated variables and disturbances are small. Secondly, their implementation error is higher than the maximum optimal change in the stage temperature, $\max \left[c_{\text {opt }}\left(d^{*}\right)-c_{\text {opt }}(d)\right], d \in D$, rendering stage temperatures useless for control. The influence of the stage temperature implementation error on the estimated loss can be emphasized by investigating the disturbance range. This is executed by multiplying a factor $r_{d}$ to Eq. 66 and varying it (ELM). If the influence of disturbances on the loss is predominant $(\nu(d)>>n)$, then the singular value of $M$ in Eq. 77) will change linearly with $r_{d}$. If implementation errors have a considerable influence $(\nu(d) \approx n)$, the change will be nonlinear. Fig. 4 shows the result for a few selected sets: The constant stage temperature implementation error gains influence for $r_{d}<1.2, r_{d} \rightarrow 0$ 
(Sets 8,16 ) while the contribution of the relative implementation error of mass flows and mass flow ratios to the worst-case loss stays always the same.

[Figure 5 about here]

Both screening methods suggest using the bottom stage temperature $T_{B 4}$ or the boilup ratio $\tilde{\nu}_{4}$ as substitute variable for the control of the product compositions $w_{B 4, C 0}$. However, the screening was conducted assuming that the vapour mass flow rates can be measured as precisely as the liquid mass flow rates. In reality, this is not the case and the measurement of boilup ratios is subject to large uncertainties. Finally it can be concluded that the sump temperature $T_{B 4}$ is the most promising substitute for the product composition $w_{B 4, C 0}$. As last step of the screening procedure, an RGA analysis is conducted. The relative gain array for Set 1 is given in Table 9. It shows that simultaneous feedback control of the distillate composition $w_{D, C 0}$ and the bottom stage temperature $T_{B 4}$ introduces serious interaction. This is a general problem posed by the process for SISO control strategies, and thus, no substitute variable in the physical proximity of the distillate stream $D$ can improve it.

[Table 10 about here]

It is concluded from an extensive screening and process insight that the reboiler heat duty should rather be adjusted using feedforward than using feedback control. The losses implied by this control strategy are evaluated exactly using nonlinear simulations in the next step.

Step 3.5 Exact evaluation of loss. Feedforward control of the reboiler heat duty is only applicable if the disturbance variables can be measured. This is possible in case of the feed mass flow rate and the feed temperature. However, there is no available online measurement of the feed quality. Therefore, a sufficient backoff has to be added to the nominal reboiler heat duty $\dot{Q}_{R B 1}^{*}$ and reflux ratio $\nu_{3}^{*}$ in order to keep the product specifications for the selected disturbance space $D$. The exact nonlinear losses $L$ imposed by this control strategy is compared in Fig. 5 and Table 7 which show that using backoffs introduces a nominal loss $L^{*}$ (disturbance scenario 0 ).

[Figure 6 about here] 
Moreover, it can be seen in Table 7 that the reboiler heat duty backoff is mainly required in order to keep the product specifications for disturbance scenario $7\left(w_{F, C 2}+900 \mathrm{ppm}\right)$.

In general, the magnitude of a backoff is always a trade-off between robustness toward process constraints and the imposed loss $L$. For this evaluation, it was assumed that the product compositions are hard constraints and may absolutely not be violated. However, depending on the distribution of the disturbance variable $w_{F, C 2}$, it could be possible to decrease the nominal reboiler heat duty backoff.

Step 3.6 Selection of controlled variables and summary. Based on the results of the candidate controlled variable screening and the nonlinear evaluation of loss, the following set of primary controlled variables is selected:

$\begin{array}{llllll}\nu_{1} & \nu_{2} & \nu_{3} & T_{B 4} & T_{D 4} & \dot{Q}_{R B 1}\end{array}$

The reboiler heat duty is adjusted with respect to the feed temperature and feed mass flow rate disturbances.

Step 4:Selection of throughput manipulator location. In order to conclude the top-down analysis, the production rate limiting process variable needs to be identified. This can be investigated rigorously using the process model and maximizing its throughput. The process variable which hits its constraint first should be used to control the production rate.

However, the heat-integrated column sequence is part of a large scale chemical plant and its throughput is determined by the production rate of upstream units. Consequently, throughput changes are defined as disturbances for the control problem and the number of steady state control degrees of freedom of the system is reduced by one.

[Figure 7 around here]

Step 5: Selection of secondary controlled variables $y_{2}$ and regulatory layer. With given inputs and controlled outputs, the objective of the bottom-up design is to find the corresponding pairing. By a given feed, 19 control degrees of freedom are still available. The pairing is conducted successively for all control layers. 
The regulatory layer has the smallest time scale of all control layers (seconds) and directly uses the physical inputs $u$ of the process for manipulation. Moreover, pressure control is vital for stabilization. For the analysis, it was assumed that the top pressures of all four columns are perfectly controlled.

Inventory control structures should be designed such that throughput changes

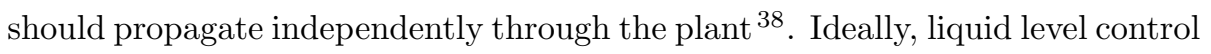
loops should be radiated outward using the throughput manipulator location as a starting point. For the heat-integrated column sequence, changes in the production rate are determined by upstream process units. Therefore, the "push" strategy should be implemented, i.e. all reflux drum levels $l_{D, i}$ are controlled with liquid distillate streams $D_{i}$, all sump levels $l_{B, i}$ are controlled with bottom streams $B, i$, and the distillate drum level $l_{\text {Drum }}$ is controlled with its only outlet flow $D$.

With level and pressure control loops closed, 17 of 23 available manipulated variables are in use for process stabilization. In order to stabilize the operation completely, a feedforward control of the steam consumption is mandatory. If kept constant in the presence of feed temperature or feed flow disturbances, trays in the last column or even in the third column will run dry (depending on the magnitude of disturbance).

In summary, the regulatory layer stabilizes the process and conducts some minor disturbance rejection, but it does not keep the operation near the optimal point. This is the task of the supervisory layer, which is designed in the next step.

Step 6: Supervisory control layer. All the remaining manipulated variables are paired as suggested by the relative gain array (Table 9). In order to control the temperature bottom stage $T_{B 4}$, the reflux flow $\dot{m}_{L 4}$ is available as manipulated variable. Table 6 suggests that the control of temperature $T_{B 4}$ is only beneficial for feed composition disturbances, while keeping the reflux ratio $\nu_{4}$ constant is sufficient to handle feed mass flow disturbances. Therefore, a cascade is implemented in order to improve the control of the fourth column for feed mass flow disturbances. 
The final control structure is shown in Figure 6 .

[Figure 8 about here]

Step 7: Optimization layer. The evaluation shows that a major fraction of the loss $L$ is caused by the backoff of the reboiler RB1 heat duty. The most effective approach to narrow the gap to optimal control is to introduce additional measurements or soft sensors of the feed and/or product qualities. Without these, model predictive controllers and other optimizing technologies have to be tuned as conservative and robustly as the SISO control structure synthesized in this work.

Step8: Dynamic validation. Figures 7 and 8 compare how closing the temperature control (TC) loop affects the operation of the fourth column. For the throughput disturbance in Figure 7 the influence of the temperature control is negligible. Adjusting the reflux ratio $v_{4}$ is sufficient, and thus, the final control result is approximately the same. The benefit of an implemented temperature controller is evident for feed composition disturbances as the one tested in Figure 8. Even though keeping reflux ratios as constant represents already a major improvement to control with constant reflux flows, both product compositions are prone to violate their specifications. Closing the temperature control loop stabilizes the product composition $w_{B 4, C 0}$ and reduces the deviation of the product composition $w_{D, C 0}$ by adjusting the reflux ratio.

Moreover, although the reboiler heat duty is feedforward controlled, it still has a significant effect on the bottom stream $B 4$. The sensitivities of the composition $w_{B 4, C 0}$ and temperature $T_{D 4}$ to changes of the reboiler heat duty and the feed state is quite large. The benefit of the temperature controller depends strongly on the frequency of the feed disturbances. Whenever the control loop's bandwidth is smaller than that of the disturbance, the temperature controller will not be able to improve the control performance. 


\section{Conclusions}

A control structure design methodology with potential to improve the workflow has been presented and applied to an industrial process. The proposed approach has been developed so as that it can be favorably adopted in chemical process industry. The case study demonstrated the benefits: (i) The process can be run to near-optimal conditions (as far as allowed by available instrumentation and disturbances), (ii) insufficient instrumentation is highlighted by increased losses, (iii) the effort of manual adjustments in order to optimize during disturbances is reduced (operator intervention) while at the same time (iv) additional benefits of implementation of an optimization layer (e.g. Real-Time Optimization) are estimated. These features offer significant potential for narrowing the gap between model-based process design and model-based process control, and could help with the standardization of these technologies, which are normally not even considered during the process of plant engineering. Another benefit is that the methodology can be applied during early stages of plant design and thus also help with the design and implementation of model-based sensor and actor placement.

However, there are certainly a number of challenges when introducing such a new method into the plant engineering workflow. In general, the plant engineering workflow is subject to immense cost and time pressure. The focus is on meeting the plant commissioning date. The economic loss of one day of startup delay is usually much higher than the benefit achieved by the difference of running the plant one day near-optimal (self-optimizing) or ?just stable?. Also, usually every single measurement that is not absolutely required for stable operation, is at least questioned. The best practices of copying control structures from similar plants/units or using heuristics are therefore widespread and building e.g. a dynamic simulation is rare. To integrate a new method into this environment as a standard is therefore difficult. Even in cases of doubt (when heuristics don?t help and there are no similar plants to copy a tested control structure from), the control structure selection method investigated in 
this work could probably not be applied because it focuses on optimized plant operation, and not on stability, which is the main priority for plant startup.

Another challenge represents simulation models which are usually kept to a bare minimum for reasons of robustness and time/cost. This means that e.g. distillation columns are not modelled rigorously, but as simple component splitters; or reactors are only modelled as fixed conversion reactors instead of using equilibrium models. In this case, the additional modelling effort for getting a meaningful result from the control structure selection algorithm can be considerable (knock out). The proposed approach directly utilizes the existing models that are available. Relatively low modeling effort is needed by converting the steady state models to dynamic for validation and final adjustments. Moreover, in almost all cases, a new plant is optimized to generate high product yield and reduce operational costs (wastes, by products, energy usage, etc.). This requires adjusting process parameters (such as- temperature, pressure, concentrations, etc.), mass and energy balances, recycle streams, heat integrations, etc. to the operating point.

From our experience, all plants (new as well as existing) can benefit that have existing steady state models which captures the causality with respect to key changes in the operating conditions- such as load changes, shift in operating conditions- disturbances/upsets, or gradual change in the process, deactivation of catalyst and by product formation.

We believe that the proposed approach will have the most potential to be integrated into the workflow, if all the secondary benefits are emphasized, as stated above. The cooperation across disciplines (engineering, control, operators, etc.) would be realistic if an optimized control structure for an existing plant is investigated. Finally, operators are the end users of the approach and are thus key beneficiary here. Basic understanding on how the plant-wide control is working is of vital importance for the success of the approach. 


\section{Appendix}

Procedure and Execution Times:

- First, update of the model in sequential modular (SM) mode and initialization of the equation-oriented (EO) mode. This needs altogether 10s (SM update is necessary to have initials defined for the EO mode).

- In EO mode, an optimization iteration takes about $0.05 \mathrm{~s}$, approximately 8 iterations are required for instance to solve one disturbance scenario, starting from the nominal operating point.

- To extract first order sensitivities (e.g. for gain or disturbance gain matrix), no extra runs are required, they are simply read from the Jacobian. Once solved in EO mode at the nominal point, the speed of extracting the sensitivities is basically determined by how fast the ASCII file can be written to the hard disk. In our case (SSD drive), it took about $0.1 \mathrm{~s}$ for each independent variable, in our case 12 independents (8 disturbance variables, 4 manipulated variables).

- As indicated in in this work, the Hessian matrix is approximated using a finite differences approach. The sensitivities are extracted at ten sample points around the nominal operating point, and then interpolated to calculate the second order sensitivities for the Hessian matrix. To reach a sample point, 1-2 iterations are needed, which take a time of around $0.05 \mathrm{~s}$ to solve 12 independents variables*2 iterations* 10 sample points* $0.05 \mathrm{~s}=$ $12 \mathrm{~s}$.

- One real-time simulated hour takes approx. 10s to be computed for the dynamic simulation.

- The branch and bound algorithm takes around 1s for the MSV rule and 5 s for the ELM rule to solve. Reading in and preparing all input data to 
the branch and bound algorithms takes about 20s though, mainly dominated by the plots for cross-checking of the quality of the finite differences approach for Hessian approximation.

- The execution time measurement was done on a laptop with i7-3517U@1,9Ghz CPU, 4GB of RAM and SSD hard drive. 


\section{Nomenclature}

\begin{tabular}{|ll|}
\hline$c$ & controlled variable (selected from the sets of $y$ and $u$ ) \\
$c_{s}$ & setpoint value for $c$ \\
$D$ & disturbance variable \\
$e_{c}$ & Disturbance space \\
$g_{1}(u, d)$ & control error \\
$g_{2}(u, d)$ & inequality constraint \\
$G$ & Gain matrix \\
$J(u, d)$ & cost function to be minimized \\
$l$ & (as superscript) lower bound \\
$L$ & Loss \\
$n$ & implementation error \\
$N$ & number \\
$o p t$ & (as subscript) optimal value \\
$u$ & manipulated variable (degrees of freedom for control) \\
$u$ & (as superscript) upper bound \\
$\nu(d)$ & setpoint error \\
$w$ & mass fraction \\
$y$ & dependent measurement process variable \\
$*$ & (mathematical notation) nominal value \\
, & (mathematical notation) normalized value \\
$\cdot$ & (mathematical notation) rate \\
\hline
\end{tabular}




\section{References}

(1) Skogestad, S. Control structure design for complete chemical plants. Comp. Chem. Eng. 2004, 28(1-2), 219-234.

(2) Buckley, P. S. Techniques of Process Control; Wiley: New York, 2004.

(3) Luyben, W.; Tyreus, B.; Luyben, M. Plantwide Process Control; McGrawHill: New York, 1999.

(4) Wolff, E.; Skogestad, S. Operability of integrated plants. In: PSE'94, Korea, 30 May 1994.

(5) Seborg, D.; Edgar, T.; Mellichap, D. Process Dynamics and Control; Wiley-Indial: New Delhi, 1989.

(6) Stichlmair, J. Conceptual design of the control configurations of distillation columns. Chem. Eng. Process. 1995, 34(2), 61-69.

(7) Luyben, W. The concept of eigenstructure in process control. Ind. Eng. Chem. Res. 1988, 27(1), 206-208.

(8) Tyreus, B. Dominant variables for partial control. Ind. Eng. Chem. Res. 1999, 38(4), 1432-1455.

(9) Stephanopoulos, G.; Ng, C. Perspectives on the synthesis of plant-wide control structures. J. Process Control. 2000, 10(2-3), 97-111.

(10) Scharf, T. Prozessbezogene optimierungsbasierte Regelungsstrukturauswahl mit Anwendungauf die Reaktivrektifikation; Lehrstuhl fr Anlagensteuerungstechnik: Universität Dortmund, 2007.

(11) Biegler, L.; Grossmann, I. Retrospective on optimization. Comput. Chem. Eng. 2004, 28(8), 1169-1192.

(12) Larsson, T.; Skogestad, S. Plantwide control-A review and a new design procedure. Modeling Identification Control. 2000, 21(4), 209-240. 
(13) Rinard, I.; Downs, J. Plant wide control: A review and critique. In: AIChE Spring Meeting 1992, New Orleans, paper 67f.

(14) van de Wal, M.; de Jager, B. Control structure design: A survey. In: Proceedings of the American Control Conference, 1995.

(15) van de Wal, M.; de Jager, B. A review of methods for input/output selection. Automatica 2001, 37(4), 487-510.

(16) Engell, S. Feedback control for optimal process operation. J. Process Control. 2007, 17(3), 203-219.

(17) Morari, M.; Arkun, Y.; Stephanopoulos, G. Studies in the synthesis of control of control structures for chemical processes. AIChE J. 1980, 26(2), 220-260.

(18) Oldenburg, J.; Pallasch, H.; Carroll, C.; Hagenmeyer, V.; Arora, S.; Jacobsen, K.; Birk, J.; Polt, A.; van den Abeel, P. Decision support for control structure selection during plant design. Comput. Aided Chem. Eng. 2008, $25,973-978$.

(19) Bausa, J.; Dunnebier, G. Life cycle modelling in the chemical industries: Is there any reuse of models in automation and control? Comput. Aided Chem. Eng. 2006, 21, 3-8.

(20) Skogestad, S. Plantwide control: The search for the self-optimizing control structure. J. Process Control. 2000, 10(5), 487-507.

(21) Skogestad, S.; Postlethwaite, I. Multivariable feedback control: Analysis and design; Wiley: New York, 1996.

(22) Halvorsen, I.; Skogestad, S.; Morud, J.; Alstad, V. Optimal selection of controlled variables. Ind. Eng. Chem. Res 2003, 42, 3273-3284.

(23) Hori, E.; Skogestad, S. Selection of controlled variables: Maximum gain rule and combination of measurements. Ind. Eng. Chem. Res 2008, 47(23), 9465-9471. 
(24) Bristol, E.. On a new measure of interaction for multivariable process control. IEEE Trans. Automatic Control 1966, 11(1), 133-134.

(25) Cao, Y.; Kariwala, V. Bidirectional branch and bound for controlled variable selection: Part I. Principle and minimum singular value criterion. Comp. Chem. Eng. 2008, 32(10), 2306-2319.

(26) Kariwala, V.; Cao, Y. Bidirectional branch and bound for controlled variable selection: Part II. Exact local method for self-optimizing control. Comp. Chem. Eng. 2009, 33(8), 1402-1412.

(27) Kariwala, V.; Cao, Y. Bidirectional branch and bound for controlled variable selection: Part III. Local average loss optimization. IEEE Trans. Ind. Informatics 2010, 6(1), 54-61.

$\begin{array}{lllll}\text { (28) Cao, } & \text { Y.; } & \text { Kariwala, } & \text { V. } & \text { B3MSV }\end{array}$ http://mathworks.com/matlabcentral/fileexchange/17480, 2009.

(29) Cao, Y.; Kariwala, V. B3WC. Improved code retrieved by personal communication., http://mathworks.com/matlabcentral/fileexchange/22632, 2009.

(30) Cao, Y.; Kariwala, V. B3AV. Improved code retrieved by personal communication., http://mathworks.com/matlabcentral/fileexchange/25870, 2010.

(31) Nocedal, J.; Wright, S. Numerical optimization; Springer Verlag: Berlin, 2006.

(32) Ponton, J. Degrees of freedom analysis in process control. Chem. Eng. Sci. 1994, 49(13), 2089-2095.

(33) Konda, N. M.; Rangaiah, G.; Krishnawamy, P. A simple and effective procedure for control degrees of freedom. Chem. Eng. Sci. 2006, 61(4), 1184-1194. 
(34) Kariwala, V. Optimal measurement combination for local self-optimizing control. Ind. Eng. Chem. Res. 2007, 46(11), 3629-3634.

(35) Kariwala, V.; Cao, Y.; Janardhanan, S. Local self-optimizing control with average loss minimization. Ind. Eng. Chem. Res. 2008, 47(4), 1150-1158.

(36) Alstad, V.; Skogestad, S.; Hori, E. Optimal measurement combinations as controlled variables. J. Process Control. 2009, 19(1), 138-148.

(37) Heldt, S. Dealing with structural constraints in self-optimizing control engineering. J. Process Control. 2010, 20(9), 1049-1058.

(38) Price, R.; Lyman, P.; Georgakis, C. Throughput manipulation in plantwide control structures. Ind. Eng. Chem. Res. 1994, 33(5), 1197-1207. 


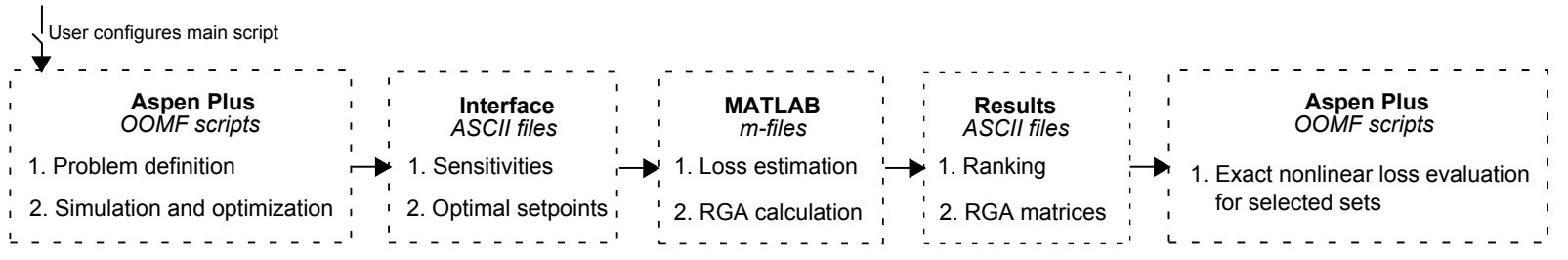

Figure 1: Tasks and data flow. 


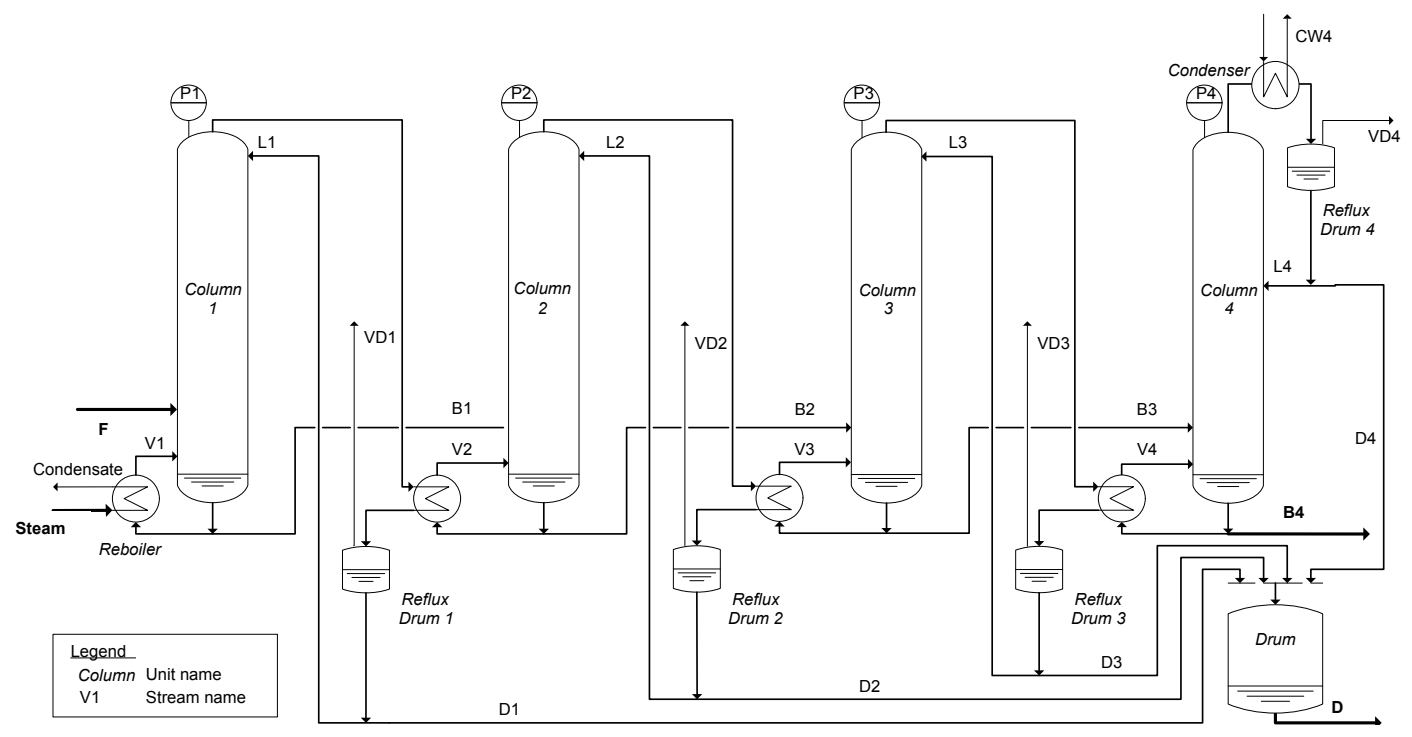

Figure 2: Heat-integrated column sequence flowsheet. 


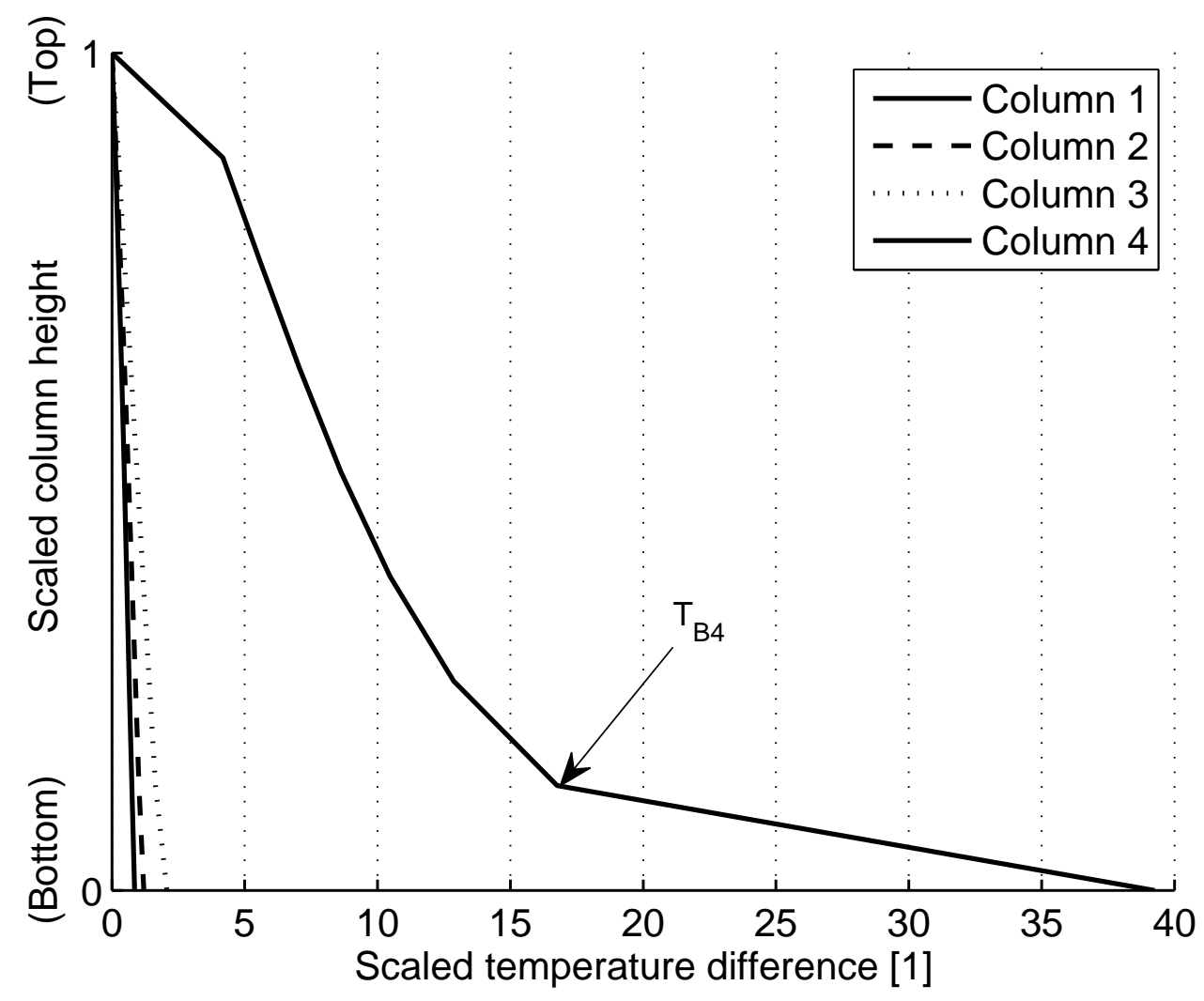

Figure 3: Nominal temperature profiles. 


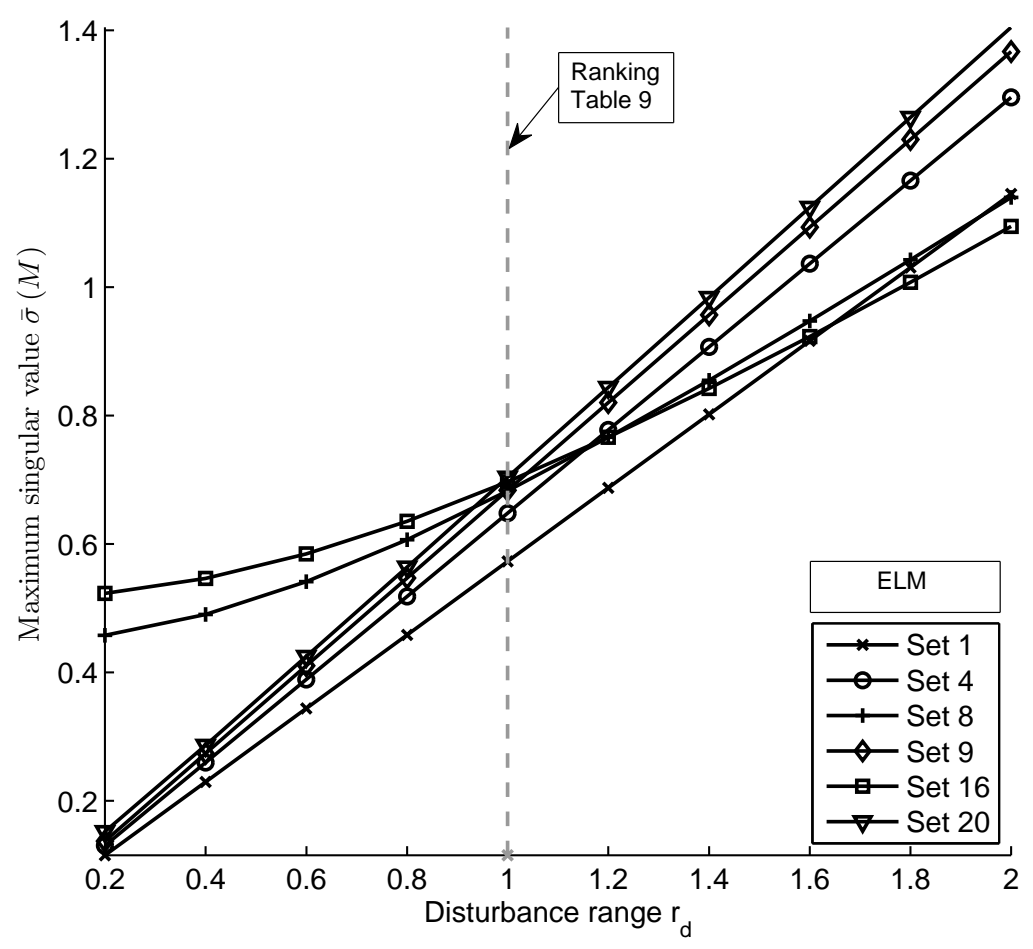

Figure 4: Maximum singular value of $M$. 


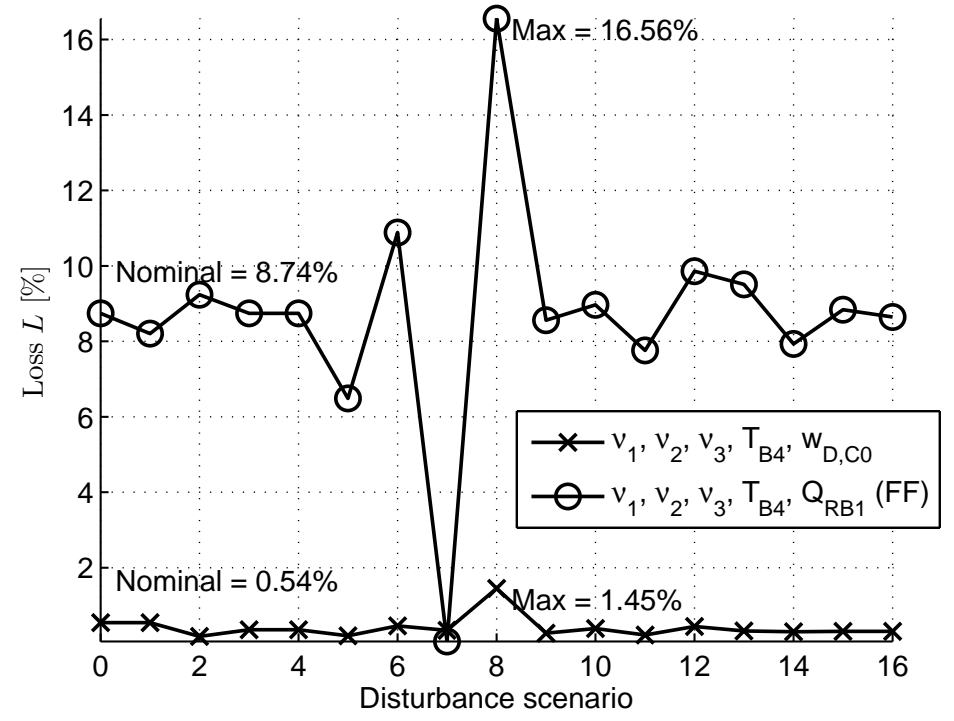

Figure 5: Comparison of exact nonlinear losses $L$. 


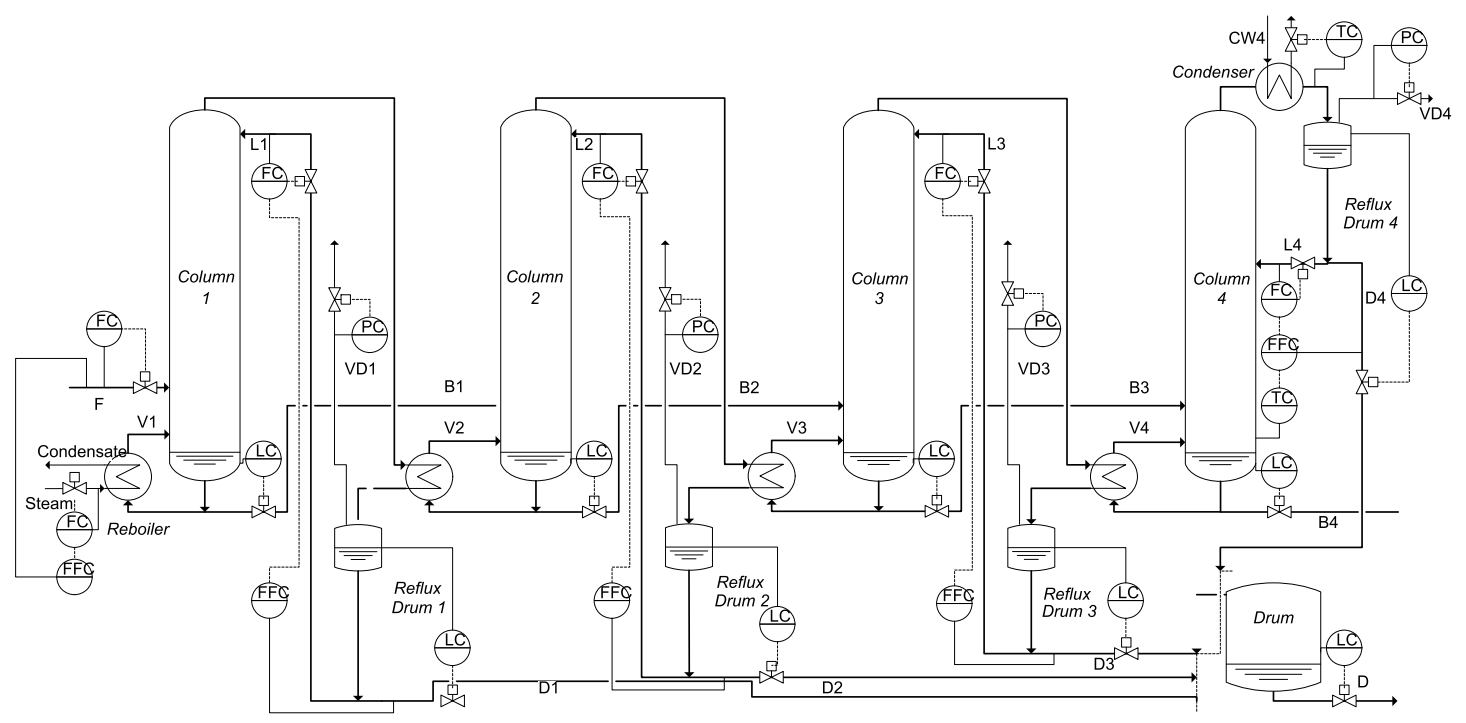

Figure 6: Final control scheme. 



Figure 7: Disturbance variable step responses. Feed mass flow decrease of $10 \%$. 

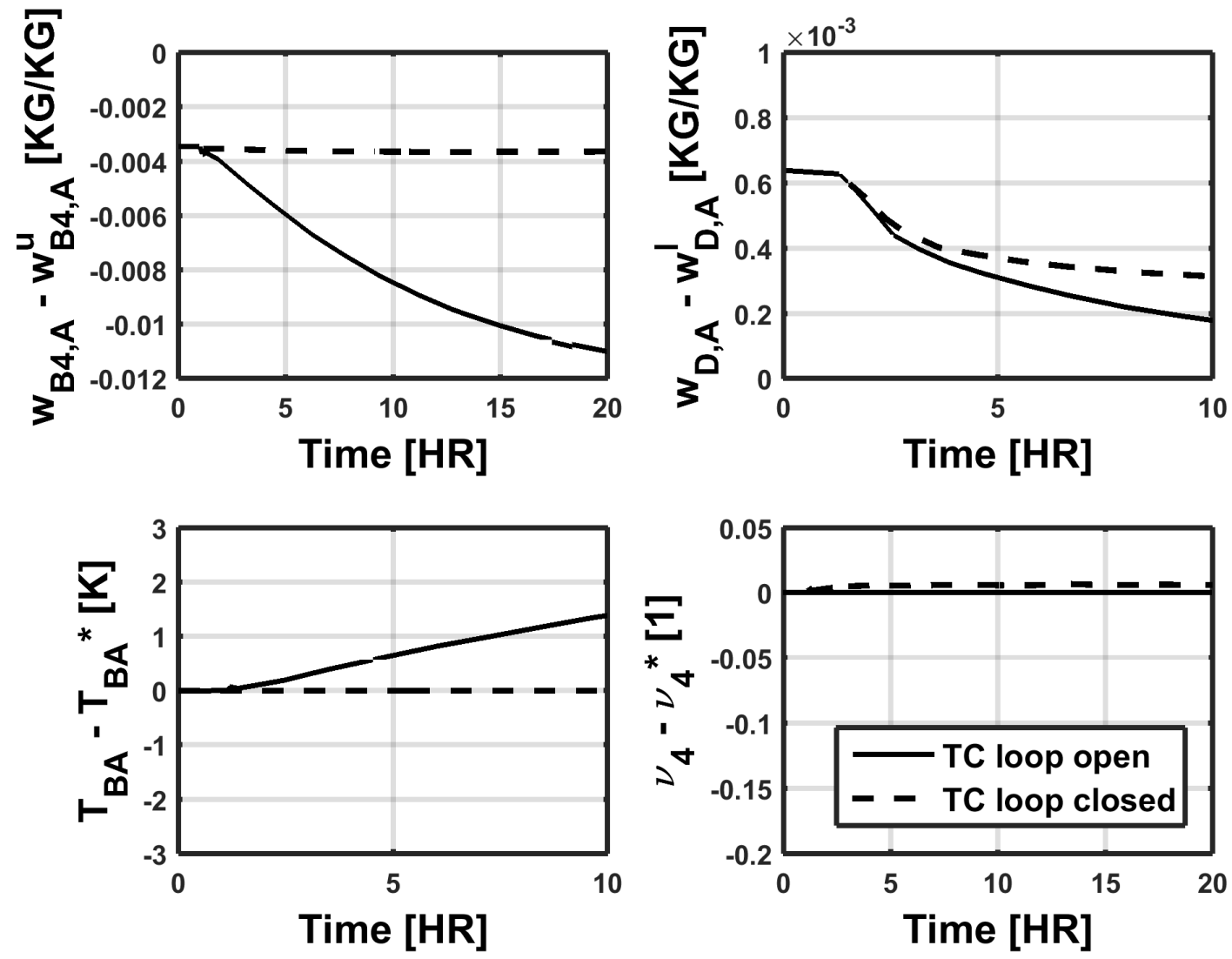

Figure 8: Disturbance variable step responses. Feed composition $w_{F, C 2}$ increase of $25 \%$. 


\begin{tabular}{lrrrrrrrr}
\hline Relative properties & \multicolumn{1}{c}{$\mathrm{i}$} & & & & & & \\
$C_{i} / C 2$ & Unit & 0 & 1 & 2 & 3 & 4 & 5 & 6 \\
\hline Boiling point & ${ }^{\circ} \mathrm{C} C$ & 0.69 & 0.91 & 1 & 1.29 & 1.30 & 1.59 & 1.85 \\
Molecular weight & & 0.24 & 1.22 & 1 & 1.03 & 2.00 & 1.81 & 2.59 \\
\hline
\end{tabular}

Table 1: Relative component properties. 


\begin{tabular}{llll}
\hline Unit & No. of units & Restraining no. & Communicating inventories \\
\hline Mixers & 1 & 1 & - \\
Splitters & 8 & 1 & - \\
Columns & 4 & 0 & - \\
Reflux drums & 4 & 0 & - \\
Distillate drum & 1 & 0 & liquid levels of reboiler \\
Reboiler 1 & 1 & 1 (hot HFP) $+2($ cold $)$ & and column sump \\
& & $1+3($ cold $)$ & cold side vapour pressure reboiler \\
Reboiler $2,3,4$ & 3 & 2 and column sump & hot side vapour pressure \\
Condenser 4 & 1 & 27 & of column and reboiler \\
\hline Overall & & & \\
\hline
\end{tabular}

Table 2: Restraining numbers. 


\begin{tabular}{lll}
\hline Manipulated variables $(u)$ & & Type \\
\hline Reboiler heat duty 1 & $\dot{Q}_{R B 1}$ & Energy stream \\
Cooling water flow 4 & $\dot{m}_{C W 4}$ & Material stream \\
Reflux flows $1,2,3,4$ & $\dot{m}_{L 1}, \dot{m}_{L 2}, \dot{m}_{L 3}, \dot{m}_{L 4}$ & Material streams \\
\hline Disturbance variables $(d)$ & & \\
\hline Feed flow & $\dot{m}_{F}$ & Material stream \\
\hline Liquid inv. control & & \\
\hline Bottom flows 1,2,3,4 & $\dot{m}_{B 1}, \dot{m}_{B 2}, \dot{m}_{B 3}, \dot{m}_{B 4}$ & Material streams \\
Distillate flows 1,2,3,4 & $\dot{m}_{D 1}, \dot{m}_{D 2}, \dot{m}_{D 3}, \dot{m}_{D 4}$ & Material streams \\
Drum outlet flow & $\dot{m}_{D}$ & Material stream $)$ \\
\hline Pressure control & & \\
\hline Vapour distillate flows $1,2,3,4$ & $\dot{m}_{V D 1}, \dot{m}_{V D 2}, \dot{m}_{V D 3}, \dot{m}_{V D 4}$ & Material streams \\
\hline
\end{tabular}

Table 3: Available manipulated variables 


\begin{tabular}{llll}
\hline $\mathrm{i}$ & Disturbance $d_{i}$ & & $d^{*}-d$ \\
\hline 1 & Feed mass flow rate & $\dot{m}_{F}$ & $40 \%$ \\
2 & Feed temperature & $T_{F}$ & $10 \mathrm{~K}$ \\
3 & Feed $C 1$ mass fraction & $w_{F, C 1}$ & $200 \mathrm{ppm}$ \\
4 & Feed $C 2$ mass fraction & $w_{F, C 2}$ & $900 \mathrm{ppm}$ \\
5 & Feed $C 3$ mass fraction & $w_{F, C 3}$ & $2000 \mathrm{ppm}$ \\
6 & Feed $C 4$ mass fraction & $w_{F, C 4}$ & $1000 \mathrm{ppm}$ \\
7 & Feed $C 5$ mass fraction & $w_{F, C 5}$ & $1000 \mathrm{ppm}$ \\
8 & Feed $C 6$ mass fraction & $w_{F, C 6}$ & $100 \mathrm{ppm}$ \\
\hline
\end{tabular}

Table 4: Disturbance variables $d_{i}$ and maximum expected perturbations $\left(d^{*}-d\right)$. 


\begin{tabular}{lcl}
\hline $\mathrm{j}$ & & $n_{j}$ \\
\hline Pressure & $\mathrm{P}$ & $0.005 \mathrm{bar}$ \\
Temperature & $\mathrm{T}$ & $0.1 \mathrm{~K}$ \\
Mass flow & $\dot{m}$ & $0.01 \dot{m}$ \\
Heat duty & $\dot{Q}$ & $0.01 \dot{Q}$ \\
Mass flow ratios & $\frac{\dot{m}_{1}}{\dot{m}_{2}}$ & $0.01 \frac{\dot{m}_{1}}{\dot{m}_{2}}$ \\
\hline
\end{tabular}

Table 5: Maximum implementation errors $n_{j}$ for process variables of physical type $\mathrm{j}$. 


\begin{tabular}{|c|c|c|c|c|c|c|c|c|}
\hline \multicolumn{9}{|l|}{ Disturbance } \\
\hline scenario & 1 & 2 & 3 & 4 & 5 & 6 & 7 & 8 \\
\hline$d_{i}$ & $\dot{m}_{F}$ & $\dot{m}_{F}$ & $T_{F}$ & $T_{F}$ & $w_{F, C 2}$ & $w_{F, C 2}$ & $w_{F, C 6}$ & $w_{F, C 6}$ \\
\hline Perturbation & $+20 \%$ & $-20 \%$ & $+10 \mathrm{~K}$ & $-10 \mathrm{~K}$ & +200 ppm & $-200 \mathrm{ppm}$ & $+200 \mathrm{ppm}$ & $-200 \mathrm{ppm}$ \\
\hline$\dot{m}_{L 1} / \dot{m}_{L 1}^{*}$ & 1.202 & 0.800 & 1.000 & 1.000 & 1.056 & 0.940 & 0.997 & 1.003 \\
\hline$\dot{m}_{L 2} / \dot{m}_{L 2}^{*}$ & 1.204 & 0.799 & 1.000 & 1.000 & 1.073 & 0.925 & 0.996 & 1.004 \\
\hline$\dot{m}_{L 3} / \dot{m}_{L 3}^{*}$ & 1.207 & 0.797 & 1.000 & 1.000 & 1.002 & 0.909 & 0.994 & 1.006 \\
\hline$\dot{m}_{L 4} / \dot{m}_{L 4}^{*}$ & 1.215 & 0.793 & 1.000 & 1.000 & 1.141 & 0.865 & 0.987 & 1.013 \\
\hline$\dot{Q}_{R B 1} / \dot{Q}_{R B 1}^{*}$ & 1.201 & 0.800 & 0.925 & 1.073 & 1.020 & 0.981 & 0.998 & 1.002 \\
\hline
\end{tabular}

Table 6: Optimization results. All values of manipulated variables $u$ are normalized to their nominal values $u^{*}$. 


\begin{tabular}{llllll}
\hline Set & $C V 1$ & $C V 2$ & $C V 3$ & $C V 4$ & $L_{\text {est }}[\%]$ \\
\hline 1 & $\nu_{1}$ & $\nu_{2}$ & $\nu_{3}$ & $T_{B 4}$ & 1.6158 \\
2 & $\alpha_{1}$ & $\nu_{2}$ & $\nu_{3}$ & $T_{B 4}$ & 1.6163 \\
3 & $\dot{m}_{L 1}$ & $\nu_{2}$ & $\nu_{3}$ & $T_{B 4}$ & 1.6164 \\
4 & $\nu_{1}$ & $\dot{m}_{L 2}$ & $\nu_{3}$ & $T_{B 4}$ & 1.6164 \\
5 & $\nu_{1}$ & $\alpha_{2}$ & $\nu_{3}$ & $T_{B 4}$ & 1.6164 \\
6 & $\alpha_{1}$ & $\dot{m}_{L 2}$ & $\nu_{3}$ & $T_{B 4}$ & 1.6168 \\
7 & $\alpha_{1}$ & $\alpha_{2}$ & $\nu_{3}$ & $T_{B 4}$ & 1.6168 \\
8 & $\dot{m}_{L 1}$ & $\dot{m}_{L 2}$ & $\nu_{3}$ & $T_{B 4}$ & 1.6170 \\
9 & $\dot{m}_{L 1}$ & $\alpha_{2}$ & $\nu_{3}$ & $T_{B 4}$ & 1.6170 \\
10 & $\nu_{1}$ & $\nu_{2}$ & $\nu_{3}$ & $\tilde{\nu}_{4}$ & 1.6754 \\
11 & $\alpha_{1}$ & $\nu_{2}$ & $\nu_{3}$ & $\tilde{\nu}_{4}$ & 1.6878 \\
12 & $\dot{m}_{L 1}$ & $\nu_{2}$ & $\nu_{3}$ & $\tilde{\nu}_{4}$ & 1.6879 \\
13 & $\nu_{1}$ & $\dot{m}_{L 2}$ & $\nu_{3}$ & $\tilde{\nu}_{4}$ & 1.6879 \\
14 & $\nu_{1}$ & $\alpha_{2}$ & $\nu_{3}$ & $\tilde{\nu}_{4}$ & 1.6891 \\
15 & $\alpha_{1}$ & $\dot{m}_{L 2}$ & $\nu_{3}$ & $\tilde{\nu}_{4}$ & 1.7024 \\
16 & $\alpha_{1}$ & $\alpha_{2}$ & $\nu_{3}$ & $\tilde{\nu}_{4}$ & 1.7024 \\
17 & $\dot{m}_{L 1}$ & $\dot{m}_{L 2}$ & $\nu_{3}$ & $\tilde{\nu}_{4}$ & 1.7039 \\
18 & $\dot{m}_{L 1}$ & $\alpha_{2}$ & $\nu_{3}$ & $\tilde{\nu}_{4}$ & 1.7039 \\
19 & $\nu_{1}$ & $\nu_{2}$ & $T_{3, x}$ & $T_{B 4}$ & 2.1199 \\
20 & $\alpha_{1}$ & $\nu_{2}$ & $T_{3, x}$ & $T_{B 4}$ & 2.1224 \\
\hline & & & & &
\end{tabular}

Table 7: Results of screening with MSV rule. 


\begin{tabular}{llllll}
\hline Set & $C V 1$ & $C V 2$ & $C V 3$ & $C V 4$ & $L_{\text {est }}[\%]$ \\
\hline 1 & $\nu_{1}$ & $\nu_{2}$ & $\nu_{3}$ & $T_{B 4}$ & 0.5580 \\
2 & $\nu_{1}$ & $\alpha_{2}$ & $\nu_{3}$ & $T_{B 4}$ & 0.6458 \\
3 & $\alpha_{1}$ & $\nu_{2}$ & $\nu_{3}$ & $T_{B 4}$ & 0.6479 \\
4 & $\nu_{1}$ & $\dot{m}_{L 2}$ & $\nu_{3}$ & $T_{B 4}$ & 0.7141 \\
5 & $\dot{m}_{L 1}$ & $\nu_{2}$ & $\nu_{3}$ & $T_{B 4}$ & 0.7182 \\
6 & $\alpha_{1}$ & $\alpha_{2}$ & $\nu_{3}$ & $T_{B 4}$ & 0.7374 \\
7 & $\nu_{1}$ & $\nu_{2}$ & $\alpha_{3}$ & $T_{B 4}$ & 0.7408 \\
8 & $\nu_{1}$ & $\nu_{2}$ & $T_{3, x}$ & $T_{B 4}$ & 0.7927 \\
9 & $\alpha_{1}$ & $\dot{m}_{L 2}$ & $\nu_{3}$ & $T_{B 4}$ & 0.7947 \\
10 & $\dot{m}_{L 1}$ & $\alpha_{2}$ & $\nu_{3}$ & $T_{B 4}$ & 0.7947 \\
11 & $\nu_{1}$ & $\alpha_{2}$ & $T_{3, x}$ & $T_{B 4}$ & 0.8024 \\
12 & $\alpha_{1}$ & $\nu_{2}$ & $T_{3, x}$ & $T_{B 4}$ & 0.8032 \\
13 & $\alpha_{1}$ & $\alpha_{2}$ & $T_{3, x}$ & $T_{B 4}$ & 0.8155 \\
14 & $\nu_{1}$ & $\nu_{2}$ & $T_{3, x}$ & $T_{B 4}$ & 0.8203 \\
15 & $\nu_{1}$ & $\alpha_{2}$ & $T_{3, x}$ & $T_{B 4}$ & 0.8252 \\
16 & $\alpha_{1}$ & $\nu_{2}$ & $T_{3, x}$ & $T_{B 4}$ & 0.8257 \\
17 & $\nu_{1}$ & $\alpha_{2}$ & $\alpha_{3}$ & $T_{B 4}$ & 0.8313 \\
18 & $\alpha_{1}$ & $\alpha_{2}$ & $T_{3, x}$ & $T_{B 4}$ & 0.8321 \\
19 & $\alpha_{1}$ & $\nu_{2}$ & $\alpha_{3}$ & $T_{B 4}$ & 0.8335 \\
20 & $\alpha_{1}$ & $\alpha_{2}$ & $\alpha_{3}$ & $\tilde{\nu}_{4}$ & 0.8429 \\
\hline & & & & &
\end{tabular}

Table 8: Results of screening with ELM. 


\begin{tabular}{l|llllll} 
& $\nu_{1}$ & $\nu_{2}$ & $\nu_{3}$ & $T_{B 4}$ & $w_{D, C 0}$ & $T_{D 4}$ \\
\hline$\dot{m}_{L 1}$ & 1 & 0 & 0 & 0 & 0 & 0 \\
$\dot{m}_{L 2}$ & 0 & 1 & 0 & 0 & 0 & 0 \\
$\dot{m}_{L 3}$ & 0 & 0 & 1 & 0 & 0 & 0 \\
$\dot{m}_{L 4}$ & 1.28 & 1.17 & 1.60 & 112.01 & -119.42 & 4.36 \\
$\dot{Q}_{R B 1}$ & -1.28 & -1.17 & -1.60 & -110.97 & 120.44 & -4.42 \\
$\dot{Q}_{C W 4}$ & 0 & 0 & 0 & -0.04 & -0.02 & 1.06
\end{tabular}

Table 9: Relative gain array for Set 1. 


\section{TOC graphic}



